\title{
Performance Analysis of OFDM Based 3-hop AF Relaying Network over Mixed Rician/Rayleigh Fading Channels
}

\author{
Praveen K. Singya ${ }^{\mathrm{a}, *}$, Nagendra Kumar ${ }^{\mathrm{b}}$, Vimal Bhatia ${ }^{\mathrm{a}}$, Senior Member, \\ IEEE and Faheem A. Khan ${ }^{c}$, Member, IEEE \\ ${ }^{a}$ Discipline of Electrical Engineering, Indian Institute of Technology Indore, India. \\ ${ }^{b}$ Department of Electronics $\&$ Communication Engineering, National Institute of \\ Technology Jamshedpur, India. \\ ${ }^{c}$ School of Computing and Engineering, University of Huddersfield, UK.
}

\begin{abstract}
In this paper, performance of an orthogonal frequency division multiplexing (OFDM) based 3-hop variable-gain amplify and forward (AF) relaying network is analyzed over independent and non-identically distributed (i.n.i.d.) mixed Rician/Rayleigh fading environment. Analytical expression of outage probability is derived and diversity order of the considered system is found. Further, average symbol error rate (ASER) expressions of general order hexagonal quadrature amplitude modulation (HQAM), general order rectangular QAM (RQAM) and 32-XQAM are derived. A comparative analysis of ASER for different QAM schemes with different constellations is also presented. Ergodic capacity with optimum rate adaptation is also derived for the considered system model. Further, the impact of Rician K-factor on the performance of the considered system is highlighted. Finally, the derived analytical results are verified through MonteCarlo simulations for different signal-to-noise ratio (SNR) levels.
\end{abstract}

Keywords: OFDM, Rician fading, ASER, hexagonal QAM (HQAM), rectangular QAM (RQAM), cross QAM (XQAM), ergodic capacity.

\section{Introduction}

During the last decade, the throughput of cellular link has increased from $2 \mathrm{Mbps}$ in $3 \mathrm{G}$ systems to about $100 \mathrm{Mbps}$ in $4 \mathrm{G}$ systems (long term evolution (LTE) and WiMAX) with multiple input multiple output (MIMO) consideration [1].

5 Although 4G systems provide improved data rate with enhanced services compared to previous wireless communication systems 1, they are still unable to satisfy the ever-increasing demands of higher data rates. Recently, the focus of mobile communication research has shifted towards development of $5 \mathrm{G}$ systems, which can provide a data rate of approx. 10 Gbps [1, 2].

\footnotetext{
*Corresponding author

Email address: phd1501102023@iiti.ac.in (Praveen K. Singya)
} 
To achieve this ambitious data rate for the user equipment (UE) in future $5 \mathrm{G}$ systems, several techniques have been discussed in [1], out of which cooperative relaying is one of them. Cooperative communication exploits the spatial diversity without the use of multiple antennas as used in MIMO systems, and has the capability to provide improved link capacity with enhanced coverage 15 and spectral efficiency [3, 4, 5, 6. Cooperative communication has gained great interest from the present wireless communication standards like 3GPP LTEAdvanced, IEEE $802.16 \mathrm{j} / \mathrm{m}$ [7] and can also be viewed as one of the promising solutions for the development of $5 \mathrm{G}$ and beyond systems.

Various relaying protocols have been proposed in the literature of which 20 amplify and forward (AF), and decode and forward (DF) are the two most commonly used protocols. In AF relaying, the signal received from the source is amplified at the relay and then forwarded to the destination. However, in DF relaying, the relay first decodes the received information, re-encodes it, and transmits it to the destination. In this work, AF relaying is preferred over

25 DF due to its low computational complexity and reduced implementation cost, however, DF outperforms the AF in terms of bit error rate (BER) in high SNR regime. A detailed study of various relaying schemes and their advantages and disadvantages are described in $3,8,0,10,11,12,13$.

Further, AF relaying can be categorized into variable-gain and fixed-gain 30 relaying. The variable-gain relaying requires knowledge of the channel state information (CSI) of the previous hop to adjust constant instantaneous transmit power at the relay, however, in fixed-gain relaying, only the average fading power of the previous hop is required to control constant average transmit power at the relay. Although fixed gain relaying is cost effective and easy to deploy, variable-

35 gain relaying provides additional performance gain as compared to fixed-gain relaying, and hence, is considered in this work [14, 15].

Additionally, orthogonal frequency division multiplexing (OFDM) is also one of the promising multicarrier modulation schemes which provides spectrally efficient high data rate communication with improved robustness even in severely 40 degraded channel conditions [16, 17]. Different wireless communication standards such as IEEE 802.11a/g/n/ac, IEEE 802.16j/m, IEEE 802.22 [18, 19], 3GPP LTE-Advanced, and digital video broadcasting [20, 21, have adopted OFDM as their main modulation technique, and is also a possible modulation scheme for $5 \mathrm{G}$ standard [22].

Thus, cooperative communication in conjunction with OFDM provides improved spectral efficiency, greater coverage and capacity enhanced link reliability and robustness at high data-rate, which provides a solid platform for future wireless communication systems 23 .

For further data-rate improvement, higher order modulation schemes such so as family of quadrature amplitude modulation (QAM) (i.e. squared QAM (SQAM), rectangular QAM (RQAM), cross QAM (XQAM) and hexagonal QAM $(\mathrm{HQAM})$ ) has gained considerable interest in present wireless communication systems for instance LTE-Advanced and in future 5G systems due to its high power and bandwidth efficiency. RQAM is a versatile modulation scheme as it 5 includes SQAM, orthogonal binary frequency shift keying (OBFSK), quadrature phase shift keying (QPSK) and multi-level amplitude shift keying (ASK) as its special cases [24. However, RQAM is not a good choice for odd number of constellation points, and an optimum XQAM constellation is preferred due to its lower peak and average power. To form a XQAM constellation, outer 
corner points of the RQAM constellation are removed and arranged in such a manner that the peak and average power of the constellation is reduced. With this, XQAM provides at-least $1 \mathrm{~dB}$ SNR gain over the RQAM scheme [25].

The increasing demand for high-data rates leads us towards the formation of optimum two dimensional (2D) hexagonal lattice based constellation referred

65 to as HQAM. HQAM has the densest 2D packing with optimum Euclidean distance between the points even for the higher order constellations along with lower peak to average power ratio (PAPR) and provides considerable SNR gain over the other QAM schemes [25, 26].

The considered system model is inspired by the shared UE-side distributed 70 antenna system (SUDAS) infrastructure [27, where end-to-end high data rate communication between two rooms of a building is established over a mixed fading environment in three time phases (3-hop). The considered system model can also be used in such cases where the source $(S)$ and destination $(D)$ are far apart, and the communication link between them is established via multiple 75 relay nodes (such as repeaters in cellular communication). Another possible use of the considered model is to increase coverage in an corporate or university campus, or for device to device (D2D) or machine to machine (M2M) communication between $S$ and $D$. The same model can be applied and extended for vehicular communication where two vehicles are equipped with on-board units

80 (OBU) and both the OBUs are connected through a ultra high frequency (UHF) link. With this, high data-rate communication can be established for the users in the vehicles by considering a channel of strong LoS component (i.e. Rician channel) between the users of the vehicle and OBU. A similar structure is also explained in [27. Thus, an OFDM based 3-hop variable-gain mixed AF relaying

85 network is considered which can be used in such cases.

In the literature, considerable work is reported on the performance analysis of a cooperative relaying network with independent and non-identically distributed (i.n.i.d.) mixed wireless links. In 28, various performance measures like outage probability and the average error probability for coherent and non90 coherent modulations are obtained, for a multi-hop cooperative network with blind relay over generalized i.n.i.d. fading channels. However, analytical results for the exact and lower-bound (LB) of the outage probability and average bit error rate $(\mathrm{ABER})$ are derived in 29 , for a dual-hop AF cooperative relaying network over mixed Rician and Rayleigh distributed links. In [30, analytical results for the outage probability and ABER are derived, for a dual-hop cooperative network for asymmetric fading environment comprising Rayleigh and Rician fading channels. However, performance of the dual-hop cooperative network is carried out in 31, over mixed Rician and Nakagami-m fading environment. Further, in [32, various performance measures such as outage probability, average symbol error probability, and ergodic capacity are derived for the dual-hop cooperative variable-gain AF relaying network, over mixed Nakagami$\mathrm{m} /$ Rician fading links. In 33, comparative analysis of BER for the OFDM based dual-hop cooperative network is carried out for various fading channels. However, power allocation and performance analysis is carried out in 34, for a multi-hop and multi-branch cooperative AF relaying network over generalized channel models. In [35], exact ergodic capacity expression is derived for the DF cooperative relaying network with the dissimilar Rician environment. In [36, performance of the dual-hop variable-gain AF relaying network is analyzed for Rician and mixed Rician/Nakagami-m fading environment, where bottleneck of 

dual-hop cooperative AF relaying network, average error probability and outage probability expressions are derived in [37, for selection combining (SC) and maximum ratio combining (MRC) receiver over i.n.i.d. Rician fading environment. Furthermore, impact of fading parameters and number of relays is also highlighted on the same system as in 36.

Further, considerable work on the average symbol error rate (ASER) performance of various QAM schemes for different wireless relaying or non-relaying systems over various fading scenarios has been reported in the literature. In [24], for a non-relaying multi-branch selection combining (SC) receiver system, (De-QPSK) are derived over i.n.i.d. Nakagami-m fading channels. For a multirelay system, closed-form expressions of ASER for HQAM, XQAM, RQAM, $\pi / 4$-QPSK and De-QPSK are derived in 25] over i.n.i.d. Nakagami-m fading links. In [26], for a single relay AF network, closed-form expressions of outage probability, asymptotic outage probability and ASER of HQAM and RQAM schemes are derived over i.n.i.d. Nakagami-m fading links with imperfect CSI. For the general order HQAM scheme, symbol error probability (SEP) expression for a non-relaying system over Rayleigh distributed channel is derived in 38. In [39], for a non-relaying system, ASER expression of triangular QAM TQAM) (special case of HQAM) is derived over additive white Gaussian noise (AWGN) channel.

To the best of authors' knowledge, no work in the literature has analysed and discussed the performance analysis of an OFDM based 3-hop cooperative relaying network over i.n.i.d. mixed Rician/Rayleigh fading links (specially ASER expressions of HQAM, RQAM and XQAM schemes) which is the focus of this paper. The considered system model is inspired by the shared UE-side distributed antenna system (SUDAS) infrastructure 27, where end-to-end high data rate communication between two rooms of a building is established over a mixed fading environment in three time phases (3-hop). From this perspective, major contributions of this paper are as follows:

- Closed-form expression of the cumulative distribution function (CDF) for the upper-bound (UB) of the end-to-end SNR, or equivalently, LB of the outage probability is obtained.

- Asymptotic analysis on the LB of the outage probability is carried out in high SNR regime.

- Precise expression for the probability density function (PDF) of the endto-end SNR is derived from the LB of the outage probability.

- Based on CDF approach, ASER expressions for general order HQAM, general order RQAM and 32-XQAM schemes are derived and comparative analysis between various QAM schemes with different constellations is presented.

- Analytical expression for the ergodic capacity using optimum rate adaptation with fixed transmission power is obtained for the considered system.

- Impact of the Rician K-factor on the performance of the relaying network is also highlighted. 
Rest of the paper is organized as follows. Section 2 describes the system and channel models for the OFDM based 3-hop variable-gain AF relaying network. Closed-form expression for the LB of the outage probability is obtained in Section 3, and asymptotic analysis on the outage probability is carried out in Section 4. In Section 5, analytical expressions of ASER for general order HQAM, general order RQAM and 32-XQAM schemes are derived. Based on PDF approach, ergodic capacity expression is derived in Section 6. In Section 7. numerical and simulation results for the proposed system are discussed, and finally, conclusions are drawn in Section 8

\section{System and Channel Models}

In this paper, an OFDM based 3-hop variable-gain AF relaying network is considered as shown in Fig. 1. Data transmission between the end users $S$ and $D$ is completed via relays $R_{1}$ and $R_{2}$. Further, no direct link between $S$ and $D$ is considered due to sufficient separation (and path loss) between the two.

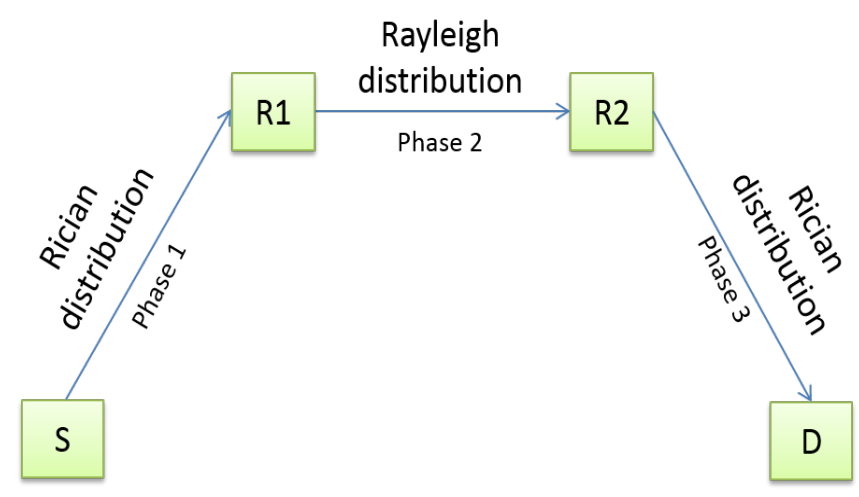

Figure 1: System model of OFDM based 3-hop AF relaying network using mixed Rician/Rayleigh/Rician wireless links.

For the considered system, end-to-end communication is completed in three orthogonal time phases, as shown in Fig. 1. In the first time phase, the first relay $\left(R_{1}\right)$ receives the signal from $S$, amplifies it using variable gain and forwards it to the second relay $\left(R_{2}\right)$ during the second phase. Received data from $R_{1}$ is amplified again by $R_{2}$ and finally forwarded to $D$ in the third phase. It is considered that $R_{1}-R_{2}$ link is Rayleigh distributed, hence, the channel gain $\left|h_{R_{1} R_{2}}\right|^{2}$ is described by a circularly symmetric complex Gaussian random variable with zero mean and $\sigma^{2}$ variance. Further, Rician distribution is considered for $S-R_{1}$ and $R_{2}-D$ links, hence, the channel gain $\left|h_{j}\right|^{2}$ (where $j=\left(s r_{1}\right.$ or $\left.r_{2} d\right)$ ) will follow the noncentral- $\chi^{2}$ distribution with two degrees of freedom. Further, length of the cyclic prefix $(\mathrm{CP})$ is considered to be greater than or equal to the channel length. All four $\left(S, R_{1}, R_{2}\right.$ and $\left.D\right)$ nodes have single antenna and are assumed synchronized at the symbol level. Further, all the nodes communicate in half-duplex mode.

Frequency domain signal received at $R_{1}$ from $S$, for the $n^{\text {th }}$ subcarrier during the first phase of communication is

$$
y_{s r_{1}}^{n}=\sqrt{P_{s}} x^{n} h_{s r_{1}}^{n}+v_{s r_{1}}^{n},
$$


where $1 \leq n \leq N$, and $N$ represents the number of subcarriers. Here, $x^{n}$ denotes the information signal for the $n^{t h}$ subcarrier, transmitted with the source power $P_{s}$. Further, $h_{s r_{1}}^{n}$ denotes the CSI for the $n^{\text {th }}$ subcarrier of the $S-R_{1}$ link, and $v_{s r_{1}}^{n}$ is the corresponding AWGN component which is represented by a circularly symmetric complex Gaussian random variable with zero mean and variance $\sigma^{2}$.

The received signal at $R_{1}$ for the $n^{t h}$ subcarrier is first amplified with the amplification factor $G_{1}^{n}$, and then transmitted to the node $R_{2}$. Thus, the signal at $R_{2}$ for the $n^{\text {th }}$ subcarrier is

$$
y_{r_{1} r_{2}}^{n}=G_{1}^{n} y_{s r_{1}}^{n} h_{r_{1} r_{2}}^{n}+v_{r_{1} r_{2}}^{n}
$$

where $h_{r_{1} r_{2}}^{n}$ denotes the CSI and $v_{r_{1} r_{2}}^{n}$ is the AWGN for the $n^{\text {th }}$ subcarrier of $R_{1}-R_{2}$ link which is also represented by circularly symmetric complex Gaussian random variable with zero mean and variance $\sigma^{2}$. Further, $G_{1}^{n}=\sqrt{\frac{P_{r_{1}}}{P_{s}\left|h_{s r_{1}}^{n}\right|^{2}+\sigma^{2}}}$ where $P_{r_{1}}$ denotes the transmit power at $R_{1}$ and $\sigma^{2}$ is the variance of the AWGN, considered to be same for each link. The CSI $\left(h_{s r_{1}}^{n}\right)$ is assumed known at the receiver.

By introducing (1) into (2), we obtain

$$
y_{r_{1} r_{2}}^{n}=G_{1}^{n} \sqrt{P_{s}} h_{s r_{1}}^{n} h_{r_{1} r_{2}}^{n} x^{n}+G_{1}^{n} h_{r_{1} r_{2}}^{n} v_{s r_{1}}^{n}+v_{r_{1} r_{2}}^{n} .
$$

Finally, $y_{r_{1} r_{2}}^{n}$ is amplified with the amplification factor $G_{2}^{n}$ at $R_{2}$, and transmitted to $D$, during the third phase of communication. Thus, at the destination, received frequency domain signal for the $n^{\text {th }}$ subcarrier is

$$
y_{r_{2} d}^{n}=G_{2}^{n} y_{r_{1} r_{2}}^{n} h_{r_{2} d}^{n}+v_{r_{2} d}^{n},
$$

where $h_{r_{2} d}^{n}$ denotes the known CSI and $v_{r_{2} d}^{n}$ is the AWGN for the $n^{\text {th }}$ subcarrier of $R_{2}-D$ link. Further, $G_{2}^{n}=\sqrt{\frac{P_{r_{2}}}{P_{r_{1}}\left|h_{r_{1} r_{2}}^{n}\right|^{2}+\sigma^{2}}}$, where $P_{r_{2}}$ is the transmit power at $R_{2}$.

After substituting (3) in (4), we obtain

$$
\begin{aligned}
y_{r_{2} d}^{n} & =G_{1}^{n} G_{2}^{n} \sqrt{P_{s}} h_{s r_{1}}^{n} h_{r_{1} r_{2}}^{n} h_{r_{2} d}^{n} x^{n}+G_{1}^{n} G_{2}^{n} h_{r_{1} r_{2}}^{n} h_{r_{2} d}^{n} v_{s r_{1}}^{n}+G_{2}^{n} h_{r_{2} d}^{n} v_{r_{1} r_{2}}^{n}+v_{r_{2} d}^{n}, \\
& =h_{s r_{1} r_{2} d}^{n} x^{n}+v_{s r_{1} r_{2} d}^{n},
\end{aligned}
$$

where

$$
\begin{aligned}
h_{s r_{1} r_{2} d}^{n} & =G_{1}^{n} G_{2}^{n} \sqrt{P_{s}} h_{s r_{1}}^{n} h_{r_{1} r_{2}}^{n} h_{r_{2} d}^{n} . \\
v_{s r_{1} r_{2} d}^{n} & =G_{1}^{n} G_{2}^{n} h_{r_{1} r_{2}}^{n} h_{r_{2} d}^{n} v_{s r_{1}}^{n}+G_{2}^{n} h_{r_{2} d}^{n} v_{r_{1} r_{2}}^{n}+v_{r_{2} d}^{n} .
\end{aligned}
$$

Hence, variance of the end-to-end link $S-R_{1}-R_{2}-D$ is

$$
\sigma_{o}^{2}=\sigma^{2}\left[1+\left(G_{2}^{n}\right)^{2}\left|h_{r_{2} d}^{n}\right|^{2}+\left(G_{1}^{n}\right)^{2}\left(G_{2}^{n}\right)^{2}\left|h_{r_{1} r_{2}}^{n}\right|^{2}\left|h_{r_{2} d}^{n}\right|^{2}\right] .
$$

Thus, instantaneous SNR of $S-R_{1}-R_{2}-D$ link is

$$
\eta_{s r_{1} r_{2} d}^{n}=\frac{\left|h_{s r_{1} r_{2} d}^{n}\right|^{2}}{\sigma_{o}^{2}} .
$$


By introducing (6) and (7) in (8), we obtain

$$
\eta_{s r_{1} r_{2} d}^{n}=\frac{\left(G_{1}^{n}\right)^{2}\left(G_{2}^{n}\right)^{2} P_{s}\left|h_{s r_{1}}^{n}\right|^{2}\left|h_{r_{1} r_{2}}^{n}\right|^{2}\left|h_{r_{2} d}^{n}\right|^{2}}{\sigma^{2}\left[\left(G_{1}^{n}\right)^{2}\left(G_{2}^{n}\right)^{2}\left|h_{r_{1} r_{2}}^{n}\right|^{2}\left|h_{r_{2} d}^{n}\right|^{2}\right]+\left(G_{2}^{n}\right)^{2}\left|h_{r_{2} d}^{n}\right|^{2}+1} .
$$

The instantaneous SNR of the $S-R_{1}, R_{1}-R_{2}$ and $R_{2}-D$ links are $\eta_{s r_{1}}^{n}=$ $\frac{\left|h_{s r_{1}}^{n}\right|^{2} P_{s}}{\sigma^{2}}, \eta_{r_{1} r_{2}}^{n}=\frac{\left|h_{r_{1} r_{2}}^{n}\right|^{2} P_{r_{1}}}{\sigma^{2}}$ and $\eta_{r_{2} d}^{n}=\frac{\left|h_{r_{2} d}^{n}\right|^{2} P_{r_{2}}}{\sigma^{2}}$, respectively. Further, their average SNR values can be given as $\bar{\eta}_{s r_{1}}^{n}=\frac{\sigma_{s r_{1}}^{2} P_{s}}{\sigma^{2}}, \bar{\eta}_{r_{1} r_{2}}^{n}=\frac{\sigma_{r_{1} r_{2}}^{2} P_{r_{1}}}{\sigma^{2}}$, and $\bar{\eta}_{r_{2} d}^{n}=$ $\frac{\sigma_{r_{2} d}^{2} P_{r_{2}}}{\sigma^{2}}$, respectively, where $\sigma_{s r_{1}}^{2}, \sigma_{r_{1} r_{2}}^{2}$ and $\sigma_{r_{2} d}^{2}$ are the average powers of the $S-R_{1}, R_{1}-R_{2}$ and $R_{2}-D$ links, respectively. Substituting the amplification factors $G_{1}^{n}, G_{2}^{n}$ and individual link's SNR values in $(9)$, instantaneous SNR of $S-R_{1}-R_{2}-D$ link for the $n^{t h}$ subcarrier can be written as

$$
\eta_{s r_{1} r_{2} d}^{n}=\frac{\eta_{s r_{1}}^{n} \eta_{r_{1} r_{2}}^{n} \eta_{r_{2} d}^{n}}{\eta_{s r_{1}}^{n} \eta_{r_{1} r_{2}}^{n}+\eta_{r_{1} r_{2}}^{n} \eta_{r_{2} d}^{n}+\eta_{s r_{1}}^{n} \eta_{r_{2} d}^{n}+\eta_{s r_{1}}^{n}+\eta_{r_{1} r_{2}}^{n}+\eta_{r_{2} d}^{n}+1} .
$$

For medium-to-high SNRs, 10 can be approximated as

$$
\eta_{s r_{1} r_{2} d}^{n} \approx \frac{1}{1 / \eta_{s r_{1}}^{n}+1 / \eta_{r_{1} r_{2}}^{n}+1 / \eta_{r_{2} d}^{n}} .
$$

From [13, 40, the UB and LB of the instantaneous SNR of $S-R_{1}-R_{2}-D$ link can be given as

$$
\frac{\eta_{\min }^{n}}{3} \leq \eta_{s r_{1} r_{2} d}^{n} \leq \eta_{\min }^{n}
$$

where $\eta_{\min }^{n}=\min \left(\eta_{s r_{1}}^{n}, \eta_{r_{1} r_{2}}^{n}, \eta_{r_{2} d}^{n}\right)$.

Further, $\eta_{s r_{1} r_{2} d}^{n}$ can be represented with its UB if one of the links has very low SNR compared to other two links, or the SNR of two out of three links is very large (infinitely), and is represented by its LB if all the links have equal SNR. In this work, UB of $\eta_{s r_{1} r_{2} d}^{n}$ is considered. Thus, $\eta_{s r_{1} r_{2} d}^{n}=\eta_{\min }^{n}=$ $\min \left(\eta_{s r_{1}}^{n}, \eta_{r_{1} r_{2}}^{n}, \eta_{r_{2} d}^{n}\right)$ [40].

\section{Outage Probability}

To characterize performance of a wireless communication network, outage probability is one of the most commonly preferred performance measures. It can be defined as the probability that the instantaneous SNR of the end-to-end wireless link goes below a predefined threshold level $\left(\eta_{t h}\right)$. For the considered UB of the end-to-end SNR 12 , LB of the outage probability for the $n^{\text {th }}$ subcarrier is

$$
P_{\text {out }, L B}^{n}\left(\eta_{t h}\right)=P\left(\eta_{\min }^{n} \leq \eta_{t h}\right)=F_{\eta_{\min }^{n}}\left(\eta_{t h}\right),
$$

where $P(\cdot)$ designates a probability. Also, $F_{\eta_{(m i n)}}^{n}\left(\eta_{t h}\right)$ represents the CDF of the random variable $\left(\eta_{\min }\right)$.

Lemma 1: Analytical expression for the LB of the outage probability for the considered system is

$$
P_{\text {out }, L B}\left(\eta_{t h}\right)=1-Q_{1}\left(\sqrt{2 K_{s r_{1}}}, \sqrt{2 \Omega_{s r_{1}} \eta_{t h}}\right) e^{\frac{-\eta_{t h}}{\bar{\eta}_{1} r_{2}}} Q_{1}\left(\sqrt{2 K_{r_{2} d}}, \sqrt{2 \Omega_{r_{2} d} \eta_{t h}}\right)
$$


where $K_{j}$ represents the Rician K-factor associated with $j^{\text {th }}$ link and $\Omega_{j}=$ $\frac{\left(K_{j}+1\right)}{\bar{\eta}_{j}}$. Further, $Q_{1}(\cdot, \cdot)$ is the first order Marcum Q-function [41, (4.35)]. Also, 215 subcarrier indexing $n$ is omitted from (14) because outage probability is independent of subcarriers due to the identical subcarrier consideration [40.

Proof: See Appendix A.

\section{Asymptotic Outage Probability}

In this Section, asymptotic analysis for the derived outage probability is carried out to obtain the diversity order of the considered cooperative network.

Lemma 2: Asymptotic approximation for the LB of the outage probability is

$$
\begin{aligned}
P_{a s y m, L B}\left(\eta_{t h}\right) & \approx 1-e^{-\left(K_{s r_{1}}+K_{r_{2} d}\right)} \sum_{l=0}^{\infty} \sum_{l_{1}=0}^{\infty} \sum_{m=0}^{l} \sum_{m_{1}=0}^{l_{1}} \frac{K_{s 1_{1}}^{l}}{l !} \frac{K_{r_{2} d}^{l_{1}}}{l_{1} !} \frac{\left(K_{s r_{1}}+1\right)^{m}}{m !} \\
& \times \frac{\left(K_{r_{2} d}+1\right)^{m_{1}}}{m_{1} !} \beta^{-m_{1}}\left(\frac{\eta_{t h}}{\bar{\eta}_{s r_{1}}}\right)^{\left(m+m_{1}\right)}\left(1-\left(\xi \frac{\eta_{t h}}{\bar{\eta}_{s r_{1}}}\right)\right)
\end{aligned}
$$

where $\xi=K_{s r_{1}}+\beta^{-1}\left(1+K_{r_{2} d}\right)+\varsigma^{-1}+1$ in which $\varsigma$ and $\beta$ are finite integers. From 15, it is clear that the asymptotic outage probability depends upon the truncation factors due to the presence of the term $\bar{\eta}_{s r_{1}}^{-\left(m+m_{1}+1\right)}$. It also depends upon the Rician K-factors $K_{s r_{1}}$ and $K_{r_{2} d}$. For $K_{s r_{1}}=K_{r_{2} d}=0 \mathrm{~dB}$ (Rayleigh case) the diversity order of the system is 1 due to the presence of relay link only. For the non-zero values of $K_{s r_{1}}$ and $K_{r_{2} d}$, the outage performance improves with the increase in Rician K-factor, however, the diversity order still remains same. This is referred to as the bottleneck effect of Rician fading in a mixed fading environment which is also reported in [36].

Proof: See Appendix B.

\section{ASER Analysis}

ASER is also a useful measure for the performance analysis of wireless communication system. In this Section, ASER expression is derived for the considered system model. For a digital modulation technique, CDF based generalized ASER expression [25, 26] is

$$
P_{s}(e)=-\int_{0}^{\infty} P_{s}^{\prime}(e \mid \eta) P_{o u t, L B}(\eta) d \eta,
$$

where $P_{s}^{\prime}(e \mid \eta)$ is the first derivative of the conditional SEP $\left(P_{s}(e \mid \eta)\right)$ for the received SNR. 
Lemma 3: For the general order HQAM scheme, ASER expression can be written as

$$
\begin{aligned}
P_{s}^{H Q A M}(e) & =-\frac{1}{2} \sqrt{\frac{\alpha}{2 \pi}}\left(\tau_{c}-\tau\right)\left[\Gamma\left(\frac{1}{2}\right)\left(\frac{\alpha}{2}\right)^{-\frac{1}{2}}-\Psi \mathbb{F}\left(\frac{\alpha}{2}\right)\right] \\
& +\frac{\tau_{c}}{3} \sqrt{\frac{\alpha}{3 \pi}}\left[\Gamma\left(\frac{1}{2}\right)\left(\frac{\alpha}{3}\right)^{-\frac{1}{2}}-\Psi \mathbb{F}\left(\frac{\alpha}{3}\right)\right] \\
& -\frac{\tau_{c}}{2} \sqrt{\frac{\alpha}{6 \pi}}\left[\Gamma\left(\frac{1}{2}\right)\left(\frac{\alpha}{6}\right)^{-\frac{1}{2}}-\Psi \mathbb{F}\left(\frac{\alpha}{6}\right)\right] \\
& -\frac{2 \tau_{c} \alpha}{9 \pi}\left[\frac{3}{2 \alpha}{ }_{2} F_{1}\left(1,1 ; \frac{3}{2} ; \frac{1}{2}\right)-\Psi \mathbb{G}\left(\frac{2 \alpha}{3}, \frac{\alpha}{3}\right)\right] \\
& +\frac{\tau_{c} \alpha}{2 \sqrt{3} \pi}\left[\frac{3}{2 \alpha}{ }_{2} F_{1}\left(1,1 ; \frac{3}{2} ; \frac{3}{4}\right)-\Psi \mathbb{G}\left(\frac{2 \alpha}{3}, \frac{\alpha}{2}\right)\right] \\
& +\frac{\tau_{c} \alpha}{2 \sqrt{3} \pi}\left[\frac{3}{2 \alpha}{ }_{2} F_{1}\left(1,1 ; \frac{3}{2} ; \frac{1}{4}\right)-\Psi \mathbb{G}\left(\frac{2 \alpha}{3}, \frac{\alpha}{6}\right)\right],
\end{aligned}
$$

where $\Psi=e^{-\left(K_{s r_{1}}+K_{r_{2} d}\right)} \sum_{l=0}^{\infty} \sum_{l_{1}=0}^{\infty} \sum_{m=0}^{l} \sum_{m_{1}=0}^{l_{1}} \frac{K_{s r_{1}}^{l}}{l !} \frac{K_{r_{2} d}^{l_{1}}}{l_{1} !} \frac{\Omega_{s r_{1}}^{m}}{m !} \frac{\Omega_{r_{2} d}^{m_{1}}}{m_{1} !}, \mathbb{F}(\theta)=$ $\Gamma\left(m+m_{1}+\frac{1}{2}\right)\left(\theta+\Omega_{s r_{1}}+\Omega_{r_{2} d}+\frac{1}{\bar{\eta}_{r_{1} r_{2}}}\right)^{-\left(m+m_{1}+\frac{1}{2}\right)}$ and $\mathbb{G}\left(\phi_{1}, \phi_{2}\right)=\Gamma\left(m+m_{1}+1\right)\left(\phi_{1}+\right.$ $\left.\Omega_{s r_{1}}+\Omega_{r_{2} d}+\frac{1}{\bar{\eta}_{r_{1} r_{2}}}\right)^{-\left(m+m_{1}+1\right)}{ }_{2} F_{1}\left(1, m+m_{1}+1 ; \frac{3}{2} ; \frac{\phi_{2}}{\phi_{1}+\Omega_{s r_{1}}+\Omega_{r_{2} d}+\frac{1}{\bar{\eta}_{1} r_{2}}}\right)$.

Proof: See Appendix C.

\subsection{Rectangular $Q A M$ (RQAM) Scheme}

Lemma 4: For the general order RQAM scheme, ASER expression can be written as

$$
\begin{aligned}
P_{s}^{R Q A M}(e) & =p_{0}+q_{0}-2 p_{0} q_{0}+\frac{2 a_{0} b_{0} p_{0} q_{0}}{\pi\left(a_{0}^{2}+b_{0}^{2}\right)}\left[{ }_{2} F_{1}\left(1,1 ; \frac{3}{2} ; \frac{a_{0}^{2}}{a_{0}^{2}+b_{0}^{2}}\right)\right. \\
& \left.+{ }_{2} F_{1}\left(1,1 ; \frac{3}{2} ; \frac{b_{0}^{2}}{a_{0}^{2}+b_{0}^{2}}\right)\right]-\Psi\left[\frac{a_{0} p_{0}\left(1-q_{0}\right)}{\sqrt{2 \pi}} \mathbb{F}\left(\frac{a_{0}^{2}}{2}\right)+\frac{b_{0} q_{0}\left(1-p_{0}\right)}{\sqrt{2 \pi}} \mathbb{F}\left(\frac{b_{0}^{2}}{2}\right)\right. \\
& \left.+\frac{a_{0} b_{0} p_{0} q_{0}}{\pi}\left(\mathbb{G}\left(\frac{a_{0}^{2}+b_{0}^{2}}{2}, \frac{a_{0}^{2}}{2}\right)+\mathbb{G}\left(\frac{a_{0}^{2}+b_{0}^{2}}{2}, \frac{b_{0}^{2}}{2}\right)\right)\right] .
\end{aligned}
$$

Proof: See Appendix D.

\subsection{Cross QAM (XQAM) Scheme}

Lemma 5: For 32-XQAM scheme, ASER expression can be written as

$$
\begin{aligned}
P_{s}^{X Q A M}(e) & =\frac{1}{8}\left\{\frac{3}{2} \sqrt{\frac{\Lambda}{\pi}}\left[\Gamma\left(\frac{1}{2}\right) \Lambda^{-\frac{1}{2}}-\Psi \mathbb{F}(\Lambda)\right]+\sqrt{\frac{\Lambda}{2 \pi}}\left[\Gamma\left(\frac{1}{2}\right)(2 \Lambda)^{-\frac{1}{2}}-\Psi \mathbb{F}(2 \Lambda)\right]\right. \\
& \left.+\frac{23 \Lambda}{\pi}\left[\frac{1}{2 \pi}{ }_{2} F_{1}\left(1,1 ; \frac{3}{2} ; \frac{1}{2}\right)-\Psi \mathbb{G}(2 \Lambda, \Lambda)\right]\right\} .
\end{aligned}
$$

Proof: See Appendix E. 


\section{Ergodic Capacity}

Out of the several techniques defined in 35, 42, for the ergodic capacity analysis, optimum rate adaptation with fixed transmission power over mixed Rician and Rayleigh fading environment is adopted because of the fixed transmission power consideration at $S, R_{1}$ and $R_{2}$ nodes. According to this scheme, transmitter adapts its rate with respect to the channel conditions while considering fixed transmit power. For a $U$-hop cooperative relaying system, channel capacity (bits/s/Hz) with optimum rate adaptation can be given as [43, 44, 45,

$$
\begin{aligned}
C_{\text {opt }} & =\frac{1}{U} \mathbb{E}\left[\log _{2}(1+\eta)\right] \\
& =\frac{1}{U} \int_{0}^{\infty} \log _{2}(1+\eta) f_{\eta_{s r_{1} r_{2} d}}(\eta) d \eta,
\end{aligned}
$$

where $\mathbb{E}[\cdot]$ and $f_{\eta_{s r_{1} r_{2} d}}(\eta)$ are the statistical expectation operator and PDF of the end-to-end instantaneous SNR, respectively. Further, $\frac{1}{U}$ corresponds to the total number of time slots required for the end-to-end transmission which is directly associated with the rate loss due to the half-duplex communication [43.

Lemma 6: Analytical expression for the ergodic capacity is

$$
\begin{aligned}
C_{o p t} & =\frac{e^{-\left(K_{s r_{1}}+K_{r_{2} d}\right)}}{U \log _{e}(2)}\left[\sum _ { l = 0 } ^ { \infty } \sum _ { m = 0 } ^ { l } \sum _ { l _ { 1 } = 0 } ^ { \infty } \frac { 1 } { m ! l ! ( l _ { 1 } ^ { 2 } ) ! } \left[K_{s r_{1}}^{l} K_{r_{2} d}^{l_{1}}\left(\Omega_{s r_{1}}\right)^{m}\left(\Omega_{r_{2} d}\right)^{l_{1}+1}\right.\right. \\
& \left.+K_{s r_{1}}^{l_{1}} K_{r_{2} d}^{l}\left(\Omega_{r_{2} d}\right)^{m}\left(\Omega_{s r_{1}}\right)^{l_{1}+1}\right] \\
& \times \frac{\left(Z_{1}-1\right) !}{\mu^{Z_{1}}}\left[\sum_{r_{1}=0}^{Z_{1}-1} \frac{(-\mu)^{r_{1}}}{r_{1} !} e^{\mu} E_{1}(\mu)+\sum_{r_{2}=1}^{Z_{1}-1} \frac{1}{r_{2}} \sum_{r_{3}=0}^{r_{2}-1} \frac{\mu^{r_{3}}}{r_{3} !} e^{-\mu} \sum_{r_{4}=0}^{Z_{1}-r_{2}-1} \frac{(-\mu)^{r_{4}}}{r_{4} !} e^{\mu}\right] \\
& +\frac{1}{\bar{\eta}_{r_{1} r_{2}}} \sum_{l=0}^{\infty} \sum_{m=0}^{l} \sum_{l_{1}=0}^{\infty} \sum_{m_{1}=0}^{l_{1}} \frac{K_{s r_{1}}^{l} K_{r_{2} d}^{l_{1}}}{l !\left(l_{1}\right) ! m ! m_{1} !}\left(\Omega_{s r_{1}}\right)^{m}\left(\Omega_{r_{2} d}\right)^{m_{1}} \frac{\left(Z_{2}-1\right) !}{\mu^{Z_{2}}} \\
& \left.\times\left[\sum_{r_{1}=0}^{Z_{2}-1} \frac{(-\mu)^{r_{1}}}{r_{1} !} e^{\mu} E_{1}(\mu)+\sum_{r_{2}=1}^{Z_{2}-1} \frac{1}{r_{2}} \sum_{r_{3}=0}^{r_{2}-1} \frac{\mu^{r_{3}}}{r_{3} !} e^{-\mu} \sum_{r_{4}=0}^{Z_{2}-r_{2}-1} \frac{(-\mu)^{r_{4}}}{r_{4} !} e^{\mu}\right]\right], \quad(21)
\end{aligned}
$$

where $Z_{1}=\left(m+l_{1}+1\right), Z_{2}=\left(m+m_{1}+1\right), \mu=\left(\Omega_{s r_{1}}+1 / \bar{\eta}_{r_{1} r_{2}}+\Omega_{r_{2} d}\right)$ and $E_{1}(\mu)=\int_{1}^{\infty} \frac{1}{s} e^{-s \mu} d s$.

Proof: See Appendix F.

\section{Numerical and Simulation Results}

In this Section, analytical expressions for the outage probability, asymptotic outage probability, ASER of general order HQAM, general order RQAM and 32-XQAM schemes, and ergodic capacity derived in Sections 3, 4, 5 and 275 6. respectively are validated by simulations. The considered Rayleigh channel is modelled with 6 independent taps. Further, 64 subcarriers OFDM system 
with a $\mathrm{CP}$ of 6 sampling periods is considered. Identical noise variance $\left(\sigma^{2}\right)$ is considered for all the wireless links. We assume that equal power is available at the $S, R_{1}$ and $R_{2}$ which is equal to unity, i.e. $P_{s}=P_{r_{1}}=P_{r_{2}}=1$. Further, theoretical results for the asymptotic outage probability and ergodic capacity are shown in 15 and $(21)$, respectively which consists of two fold infinite summations, and the summands will decay faster than the exponential decay for increasing values of $l$ and $l_{1}$ because of the presence of $\frac{1}{l ! m ! l_{1} ! m_{1} !}$ factor in 15 and (21). Hence, infinite summations $l$ and $l_{1}$ are truncated up to finite values $L$ and $L_{1}$, respectively to reduce the computational complexity with considerable accuracy.

In Fig. 22 theoretical, simulation and asymptotic results for the LB of the outage probability versus transmit SNR are compared for different values of $\eta_{t h}$ while Rician K-factor $K_{j}=5 \mathrm{~dB}$ is considering for the analysis. Theoretical results for the asymptotic outage probability are obtained by fixing the truncation parameter $L=L_{1}=17$. From Fig. 2, it is observed that the theoretical curves are always below simulation curves which corroborates the LB of the outage probability. Simulation curves overlap the theoretical curves of the outage probability at medium and high SNRs which validates the derived theoretical re5 sults. Further, the asymptotic curves follow the LB of the outage probability at high SNR with significant accuracy which validates the assumption considered for the end-to-end SNR in 11.

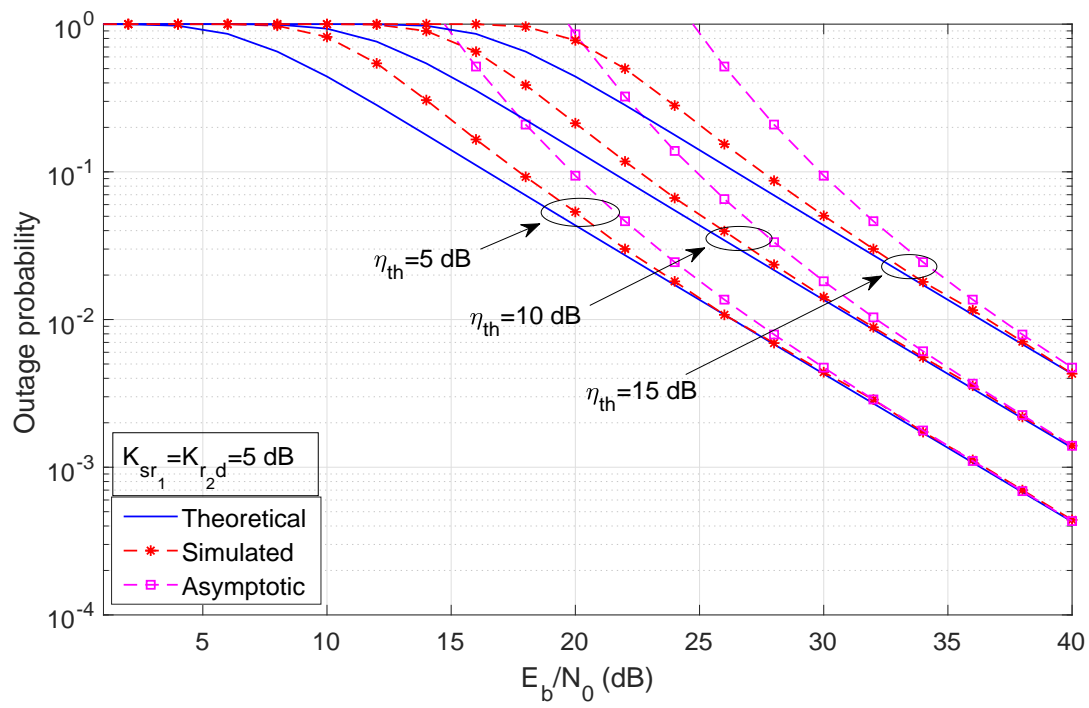

Figure 2: Comparison of theoretical, simulation and asymptotic results for the LB of the outage probability versus transmit SNR for various values of $\eta_{t h}$.

For different values of $\eta_{t h}$ and for various values of $K_{j}\left(K_{j}=0,5,9 \mathrm{~dB}\right.$ for the $j^{\text {th }}$ link), theoretical results for the LB of outage probability versus transmit SNR are shown in Fig. 3. It is observed that significant improvement in outage performance in achieved as compared to when $K_{j}=0 \mathrm{~dB}$ (equivalent to Rayleigh distribution). However, the rate of improvement decreases by further increasing the values of Rician K-factor which is clear from the cases $K_{j}=5$ $\mathrm{dB}$ and $K_{j}=9 \mathrm{~dB}$, as shown in Fig. 3. However, with the increase in $K_{j}$, 


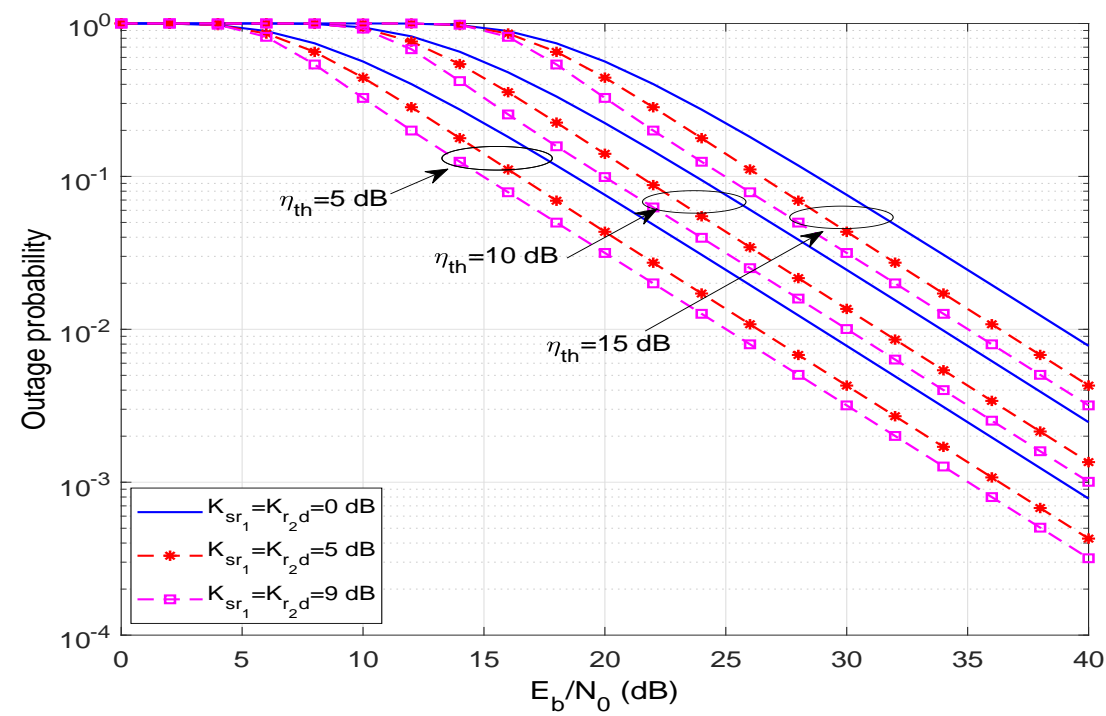

Figure 3: Comparison of theoretical results for the LB of the outage probability versus transmit SNR, for various combinations of $K_{s r_{1}}$ and $K_{r_{2} d}$ and for various values of $\eta_{t h}$.

no diversity gain is achieved because limiting slope of the outage probability curves remain unchanged irrespective of the values of Rician K-factors, which is referred to as bottleneck effect of Rician fading in a mixed fading environment [36.

In Fig. 4, theoretical and simulation results of the ASER of HQAM scheme with different constellations are presented while Rician K-factor $K_{j}=5 \mathrm{~dB}$ is considered. From the performance curves it is observed that the theoretical curves of HQAM follow the simulation curves with significant accuracy at medium and high SNRs which corroborates the derived theoretical result for the HQAM.

In Fig. 5, a comparative analysis of theoretical results of the ASER of HQAM, RQAM, XQAM and SQAM schemes with different constellations is presented while Rician K-factor $K_{j}=5 \mathrm{~dB}$ is considered. From the performance curves it is observed that the HQAM provides better ASER performance than SQAM for all the constellation orders. This is due to higher values of $\alpha$ with relatively lower PAPR than SQAM. The same is also observed in 25, 38. The rate of improvement in ASER performance for HQAM increases with the increase in constellation order. It is also observed that HQAM provides considerable improvement in ASER performance over RQAM scheme for all the constellations due to its lower PAPR than RQAM scheme. To achieve an ASER of $10^{-3}$, 4-HQAM 16-HQAM and 64-HQAM provide approx. $0.17 \mathrm{~dB}, 0.75 \mathrm{~dB}$ and $1 \mathrm{~dB}$ SNR gain over respective SQAM schemes. To achieve an ASER of $10^{-3}$, 8-HQAM and 32-HQAM provide approx. $0.8 \mathrm{~dB}$ and $1.3 \mathrm{~dB}$ SNR gain over $4 \times 2$-RQAM and $8 \times 4$-RQAM schemes. Further, for $10^{-3}$ ASER, 32-HQAM provides around $0.1 \mathrm{~dB}$ gain over 32-XQAM which provides around $1.2 \mathrm{~dB}$ gain over $8 \times 4$-RQAM. This clearly demonstrates the superiority of HQAM scheme over the other modulation schemes. 


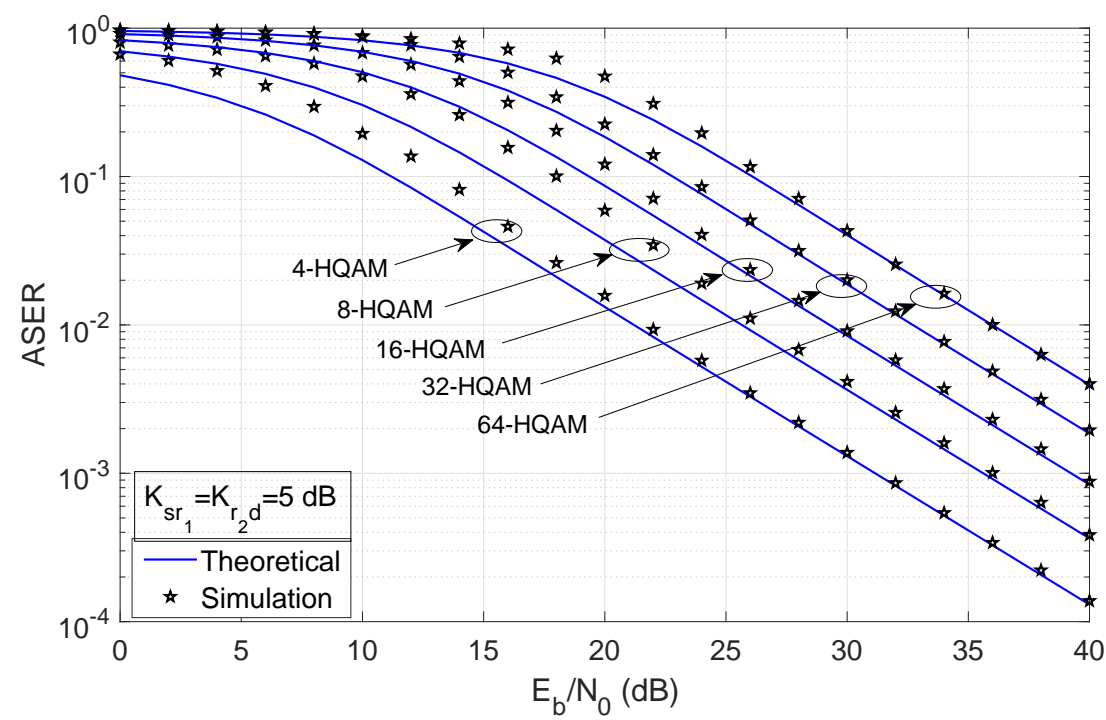

Figure 4: Comparison between theoretical and simulation results of ASER versus transmit SNR for various HQAM constellations.

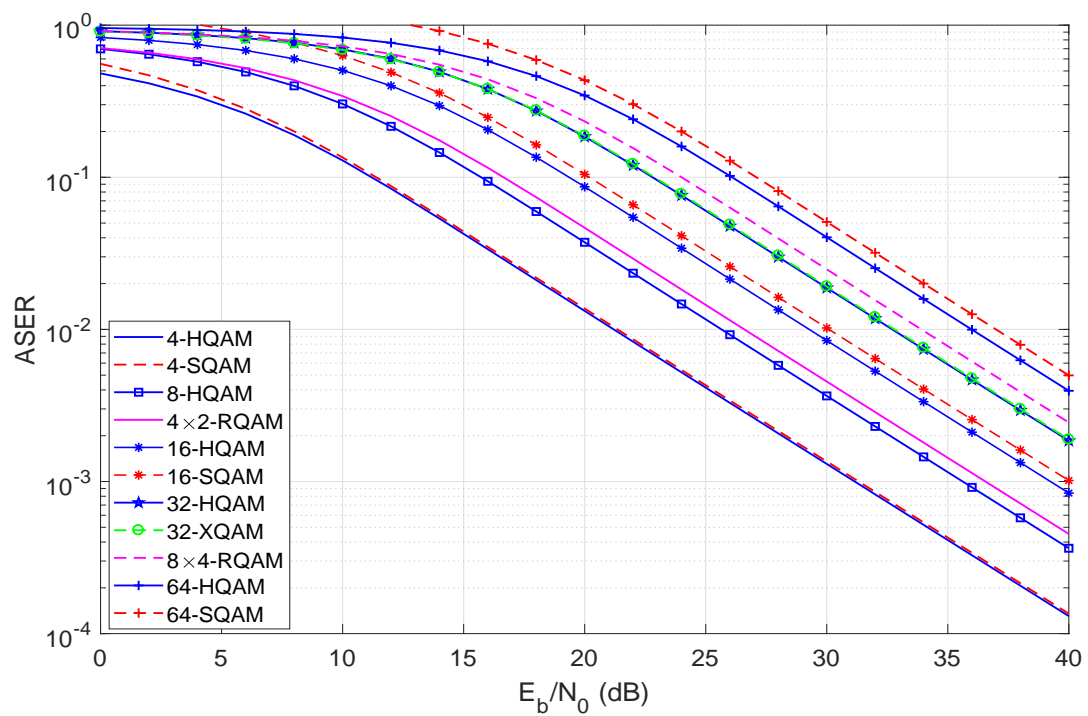

Figure 5: Comparison of theoretical results of ASER versus transmit SNR for various QAM schemes with different constellations.

For various values of $K_{j}\left(K_{j}=0,1,2,3,4,5 \mathrm{~dB}\right)$, comparative analysis for the theoretical results of the ASER of 16-HQAM is illustrated in Fig. 6. From Fig. 6, it is clear that considerable improvement in the ASER performance is achieved by increasing the values of $K_{j}$ to validate the positive impact of the Rician K-factors on the performance of the considered system.

In Fig. 7, analytical and simulated results for the ergodic capacity versus 


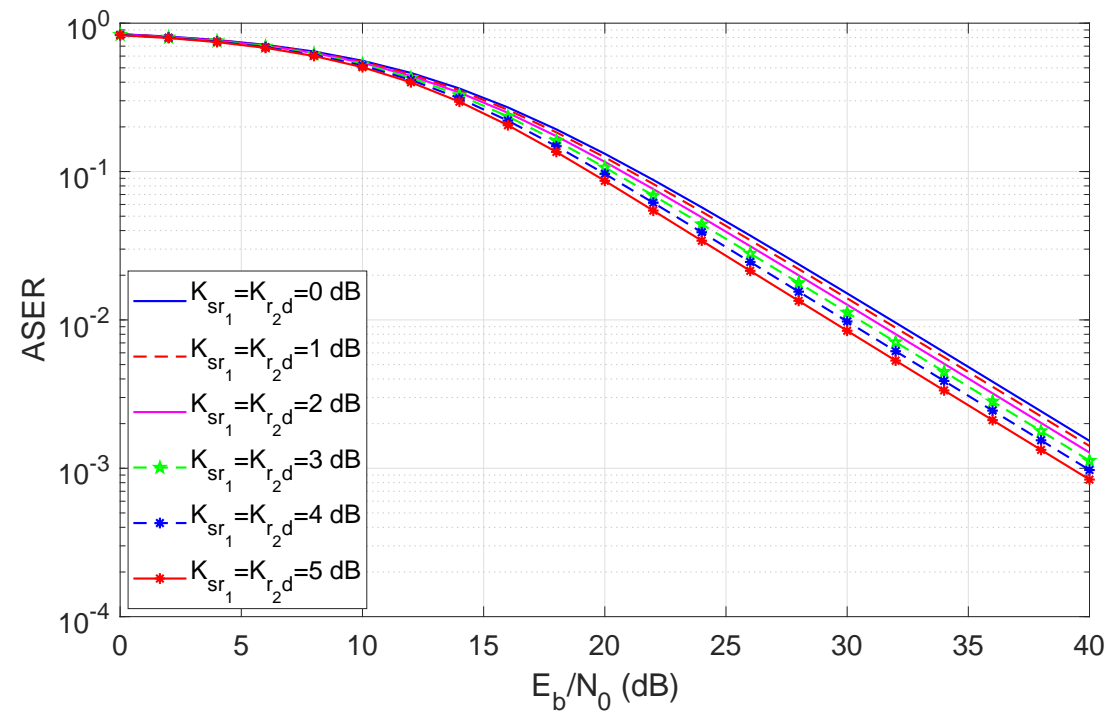

Figure 6: Comparison of theoretical results of ASER of 16-HQAM versus transmit SNR for various combinations of $K_{s r_{1}}$ and $K_{r_{2} d}$.

Table 1: Values of the ergodic capacity in 21), for different values of transmit SNR $\left(E_{b} / N_{0}\right)$ and for various combinations of the truncation parameters ( $L$ and $\left.L_{1}\right)$.

\begin{tabular}{|l|l|l|l|}
\hline \multicolumn{4}{|c|}{ Ergodic Capacity Analysis } \\
\hline$\frac{E_{b}}{N_{0}}($ in $\mathrm{dB})$ & $L=L_{1}=15$ & $L=L_{1}=17$ & $L=L_{1}=20$ \\
\hline 0 & 0.161600319129622 & 0.161600413549101 & 0.161600416652408 \\
\hline 6 & 0.429698505580225 & 0.429698743649382 & 0.429698741473835 \\
\hline 12 & 0.87711925984598 & 0.877119724225866 & 0.877119729488114 \\
\hline 18 & 1.448426277675754 & 1.448427021949692 & 1.448427026410753 \\
\hline 24 & 2.078907742183811 & 2.078908891200468 & 2.078908895676998 \\
\hline 30 & 2.732092845508648 & 2.732094208708603 & 2.732094203510869 \\
\hline 36 & 3.392990476757751 & 3.392992157341436 & 3.392992156346721 \\
\hline
\end{tabular}

transmit SNR are compared while the Rician K-factors $K_{s r_{1}}=1 \mathrm{~dB}$ and $K_{r_{2} d}=$ $2 \mathrm{~dB}$ are considered. Theoretical results for the ergodic capacity versus transmit SNR are obtained by truncating the infinite-series summations with severa combinations ( $L=L_{1}=15, L=L_{1}=17$ and $L=L_{1}=20$ ) which is shown in the Table1. From the Table, it is observed that there is no change in the ergodic capacity for up to 8 decimal places for various transmit SNRs, while $L=L_{1}$ are changed from 17 to 20 . Thus, the theoretical result for ergodic capacity is obtained by fixing the truncation parameters $L=L_{1}=17$ due to hardware limitations. From Fig. 7, it is observed that the theoretical results for the ergodic capacity overlap with the simulation results for medium and high SNR regime (after $15 \mathrm{~dB}$ both the results are overlapping exactly as also magnified in Fig. 7] which validates correctness of the obtained theoretical results for the 350 ergodic capacity at various transmit SNRs.

For various combinations of $K_{s r_{1}}$ and $K_{r_{2} d}$, comparative analysis of the 


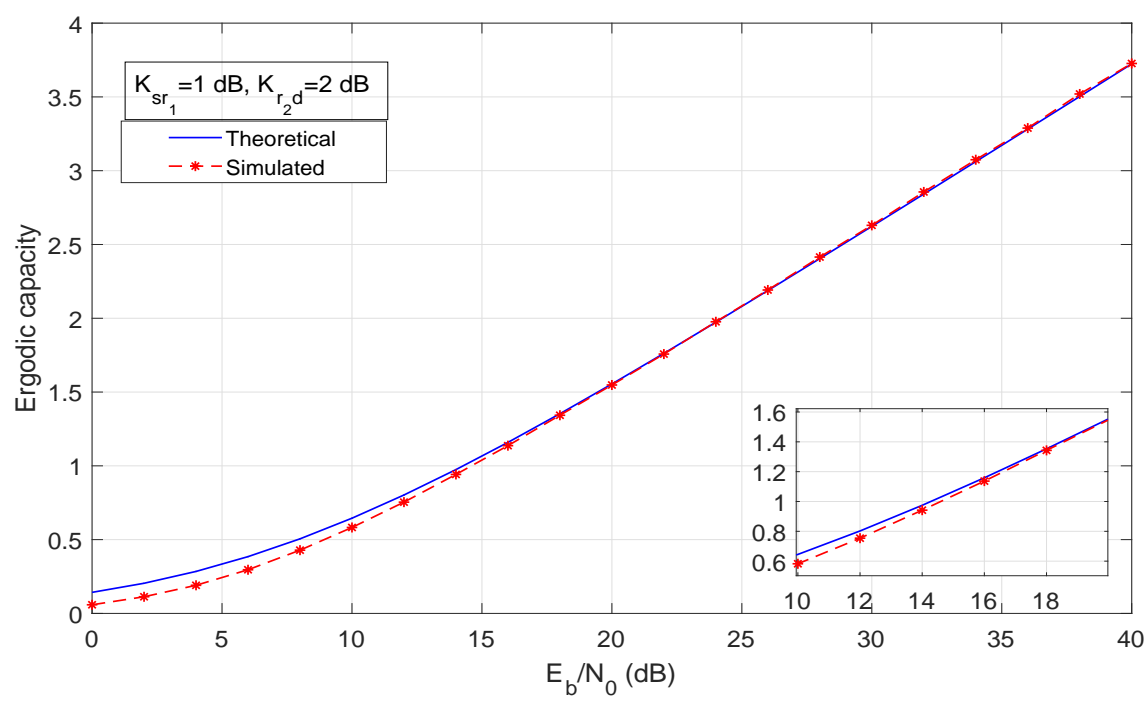

Figure 7: Comparison between theoretical and simulation results of the ergodic capacity versus transmit SNR, for $K_{s r_{1}}=1 \mathrm{~dB}$ and $K_{r_{2} d}=2 \mathrm{~dB}$.

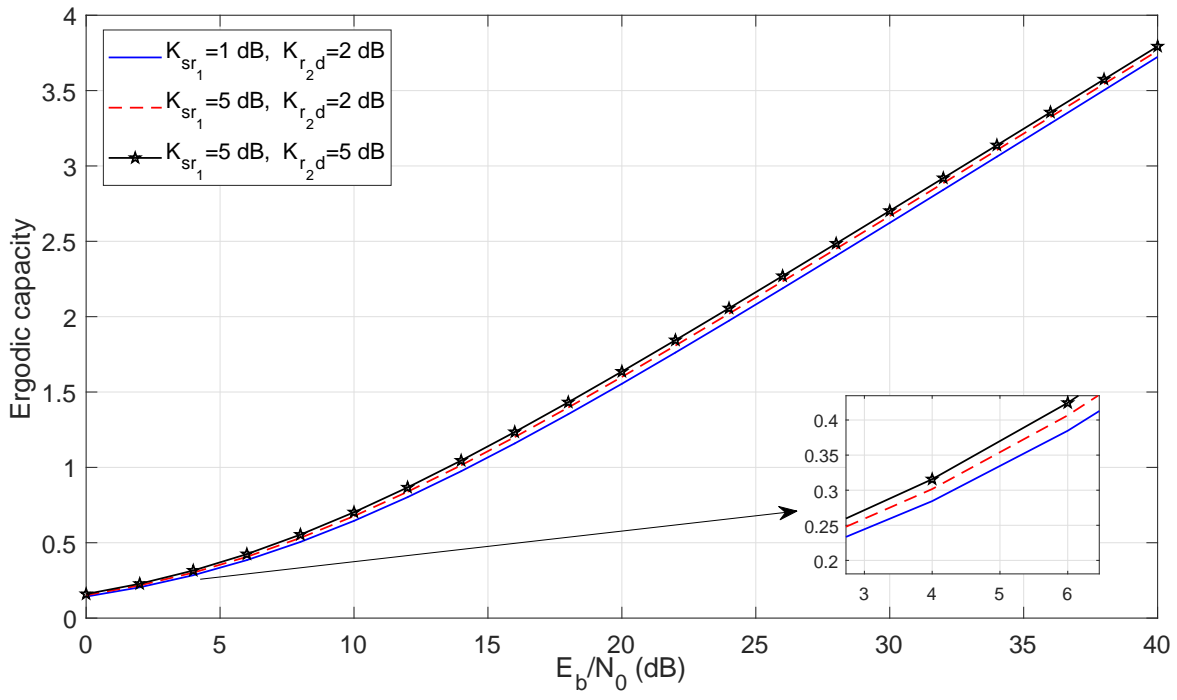

Figure 8: Comparison of the theoretical ergodic capacity versus transmit SNR, for various combinations of $K_{s r_{1}}$ and $K_{r_{2} d}$.

theoretical ergodic capacity versus transmit SNR is shown in Fig. 8. For the analysis $\left(K_{s r_{1}}=1\right.$ and $\left.K_{r_{2} d}=2\right),\left(K_{s r_{1}}=5\right.$ and $\left.K_{r_{2} d}=2\right),\left(K_{s r_{1}}=K_{r_{2} d}=5\right)$ and $L=L_{1}=17$ are considered. From Fig. 8, it is observed that the ergodic capacity of the considered system improves correspondingly with increase in the Rician K-factor of the particular link which is shown by the magnified portion in the same figure. 


\section{Conclusion}

In this paper, performance of an OFDM based 3-hop cooperative variablegain AF relaying network, over i.n.i.d. mixed Rician and Rayleigh distributed links is analyzed. Analytical expressions for the outage probability, asymptotic outage probability, ASER of general order HQAM, general order RQAM and 32-XQAM schemes, and ergodic capacity are derived. It is observed that the simulation results follow derived theoretical expressions with significant accuracy in medium and high SNR regime. From the ASER performance, it can be concluded that the HQAM scheme provides superior performance over the other modulation schemes (i.e. RQAM, XQAM and SQAM schemes). Further, considerable improvement in the performance of the outage probability, ASER and ergodic capacity is observed by increasing the values of Rician K-factors, which supports the positive impact of the Rician K-factor on the performance of the considered system. It is also observed that by increasing the value of Rician $\mathrm{K}$-factors, no diversity gain is achieved since the limiting slope of the outage probability curves remain unchanged irrespective of the values of the Rician $\mathrm{K}$-factor. The bottleneck effect of Rician fading in mixed environment is also highlighted. This work can be extended for the imperfect channel estimation and equalization in future. The analysis can be useful for $5 \mathrm{G}$ and beyond communications, and easily applied to shared UE-side distributed antenna system (SUDAS), analysis of enhance indoor coverage of mobile networks, and high speed device to device or machine-to-machine communication.

\section{Appendix A. Proof of Lemma 1}

$$
\begin{aligned}
& \text { Since } \eta_{\min }^{n}=\min \left(\eta_{s r_{1}}^{n}, \eta_{r_{1} r_{2}}^{n}, \eta_{r_{2} d}^{n}\right) \text {, its CDF is } \\
& \begin{aligned}
F_{\eta_{\min }^{n}}^{n}\left(\eta_{t h}\right) & =1-P\left(\eta_{s r_{1}}^{n} \geq \eta_{t h}\right) P\left(\eta_{r_{1} r_{2}}^{n} \geq \eta_{t h}\right) P\left(\eta_{r_{2} d}^{n} \geq \eta_{t h}\right) \\
& =1-\left[1-F_{\eta_{s r_{1}}^{n}}\left(\eta_{t h}\right)\right]\left[1-F_{\eta_{r_{1} r_{2}}^{n}}\left(\eta_{t h}\right)\right]\left[1-F_{\eta_{r_{2} d}^{n}}\left(\eta_{t h}\right)\right],
\end{aligned}
\end{aligned}
$$

where $F_{\eta_{(\cdot)}}^{n}\left(\eta_{t h}\right)$ represents the $\mathrm{CDF}$ of the SNR of $S-R_{1}, R_{1}-R_{2}$ and $R_{2}-D$ links. $S-R_{1}$ and $R_{2}-D$ links are exposed to Rician fading, and $R_{1}-R_{2}$ link is exposed to Rayleigh fading. We know that, if a link is experiencing Rayleigh fading then the channel gain $\left|h_{r_{1} r_{2}}\right|^{2}$ will be exponentially distributed random variable (RV). Hence, its PDF and CDF can be written as 46]

$$
f_{\eta_{r_{1} r_{2}}^{n}}(\eta)=\frac{1}{\bar{\eta}_{r_{1} r_{2}}} e^{\frac{-\eta}{\bar{\eta}_{1} r_{2}}}
$$

and

$$
F_{\eta_{r_{1} r_{2}}^{n}}(\eta)=1-e^{\frac{-\eta}{\bar{\eta}_{r_{1} r_{2}}}}
$$

respectively where $\bar{\eta}_{r_{1} r_{2}}$ represents the average SNR of the $R_{1}-R_{2}$ link. Also, for the Rician distributed link, the channel gain $\left|h_{j}\right|^{2}$ (where $j=\left(s r_{1}\right.$ or $\left.r_{2} d\right)$ ) will follow the noncentral- $\chi^{2}$ distribution with two degrees of freedom [30. Hence, its PDF and CDF are

$$
f_{\eta_{j}^{n}}(\eta)=\Omega_{j} e^{-\left(K_{j}+\Omega_{j} \eta\right)} I_{0}\left(2 \sqrt{K_{j} \Omega_{j} \eta}\right),
$$


and

$$
F_{\eta_{j}^{n}}(\eta)=\left(1-Q_{1}\left(\sqrt{2 K_{j}}, \sqrt{2 \Omega_{j} \eta}\right)\right)
$$

respectively, where $f(\cdot)$ and $F(\cdot)$ represent the PDF and CDF, respectively. Further, $I_{0}(\cdot)$ represents $0^{\text {th }}$ order modified Bessel function of first kind. After substituting the CDF of the particular links given in A.3) and A.5 into A.1, final expression for the LB of the outage probability is shown in (14).

\section{Appendix B. Proof of Lemma 2}

For the asymptotic analysis of the LB of the outage probability, approximate form of the Marcum Q-function is substituted in (14). Thus, by using [41, (4.47)], approximate form of the first order Marcum Q-function can be given as

$$
Q_{1}\left(\sqrt{2 K_{j}}, \sqrt{2 \Omega_{j} \eta}\right)=e^{\left(-K_{j}-\Omega_{j} \eta\right)} \sum_{l=0}^{\infty} \frac{K_{j}^{l}}{l !} \sum_{m=0}^{l} \frac{\left(\Omega_{j} \eta\right)^{m}}{m !} .
$$

By introducing (B.1) into (14), infinite-series expression for the LB of the outage probability is

$$
\begin{aligned}
P_{\text {out }, L B}\left(\eta_{t h}\right) \approx 1 & -e^{-\left(K_{s r_{1}}+K_{r_{2} d}\right)} e^{-\left(\Omega_{s r_{1}}+1 / \eta_{r_{1} r_{2}}+\Omega_{r_{2} d}\right) \eta_{t h}} \\
& \times \sum_{l=0}^{\infty} \sum_{l_{1}=0}^{\infty} \sum_{m=0}^{l} \sum_{m_{1}=0}^{l_{1}} \frac{K_{s r_{1}}^{l}}{l !} \frac{K_{r_{2} d}^{l_{1}}}{l_{1} !} \frac{\left(\Omega_{s r_{1}} \eta_{t h}\right)^{m}}{m !} \frac{\left(\Omega_{r_{2} d} \eta_{t h}\right)^{m_{1}}}{m_{1} !} .
\end{aligned}
$$

Further, considering $\bar{\eta}_{s r_{1}}, \bar{\eta}_{r_{1} r_{2}}, \bar{\eta}_{r_{2} d} \rightarrow \infty$ with $\bar{\eta}_{r_{1} r_{2}}=\alpha \bar{\eta}_{s r_{1}}$ and $\bar{\eta}_{r_{2} d}=$ $\beta \bar{\eta}_{s r_{1}}$, and with the first order exponential approximation for $(\mathrm{B} .2)$, asymptotic approximation for the outage probability is expressed in 15 .

\section{Appendix C. Proof of Lemma 3}

For M-ary HQAM scheme, conditional SEP expression in AWGN channel is

$$
P_{s}^{H Q A M}(e \mid \eta)=Q(\sqrt{\alpha \eta})\left[\tau-2 \tau_{c} Q\left(\sqrt{\frac{\alpha \eta}{3}}\right)\right]+\frac{2}{3} \tau_{c} Q^{2}\left(\sqrt{\frac{2 \alpha \eta}{3}}\right),
$$

where constants $\alpha, \tau$ and $\tau_{c}$ are used to select various HQAM constellations which are defined in [38]. Substituting the Gaussian Q-function $Q(x)=\frac{1}{2}[1-$ $\left.\operatorname{erf}\left(\frac{x}{\sqrt{2}}\right)\right]$ in $[$ C.1 and using [47, (7.1.21)], first order derivative of $($ C.1 is

$$
\begin{aligned}
P_{s}^{\prime H Q A M}(e \mid \eta)= & \eta^{-1 / 2}\left[\frac{1}{2} \sqrt{\frac{\alpha}{2 \pi}}\left(\tau_{c}-\tau\right) e^{-\frac{\alpha \eta}{2}}-\frac{\tau_{c}}{3} \sqrt{\frac{\alpha}{3 \pi}} e^{-\frac{\alpha \eta}{3}}+\frac{\tau_{c}}{2} \sqrt{\frac{\alpha}{6 \pi}} e^{-\frac{\alpha \eta}{6}}\right] \\
& +\frac{2 \tau_{c} \alpha}{9 \pi} e^{-\frac{2 \alpha}{3} \eta}{ }_{1} F_{1}\left(1 ; \frac{3}{2} ; \frac{\alpha}{3} \eta\right)-\frac{\tau_{c} \alpha e^{-\frac{2 \alpha}{3} \eta}}{2 \sqrt{3} \pi}\left[{ }_{1} F_{1}\left(1 ; \frac{3}{2} ; \frac{\alpha}{2} \eta\right)\right. \\
& \left.+{ }_{1} F_{1}\left(1 ; \frac{3}{2} ; \frac{\alpha}{6} \eta\right)\right] .
\end{aligned}
$$


Substituting $P_{s}^{\prime H Q A M}(e \mid \eta)$ and $P_{\text {out }, L B}(\eta)$ from $\sqrt{\mathrm{C} .2}$ and $\mathrm{B} .2$, respectively in (16) and solving the required integrals with the help of [48, (3.381.4), (7.522.9)], ASER expression for the general order HQAM scheme can be expressed as 17 .

Appendix D. Proof of Lemma 4

Generalized conditional SEP expression for $M_{I} \times M_{Q}$-RQAM scheme can be given as

$$
P_{s}^{R Q A M}(e \mid \eta)=2\left[p_{0} Q\left(a_{0} \sqrt{\eta}\right)\left(1-2 q_{0} Q\left(b_{0} \sqrt{\eta}\right)\right)+q_{0} Q\left(b_{0} \sqrt{\eta}\right)\right]
$$

where $p_{0}=1-\left(1 / M_{I}\right), q_{0}=1-\left(1 / M_{Q}\right), a_{0}=\sqrt{6 /\left(\left(M_{I}^{2}-1\right)+\left(M_{Q}^{2}-1\right) \lambda^{2}\right)}$, $b_{0}=\lambda a_{0} . \lambda=d_{Q} / d_{I}$ is the ratio of quadrature and in-phase decision distances. Further, $M_{I}$ and $M_{Q}$ denote the in-phase and quadrature phase constellation points, respectively. First order derivative of (D.1) can be expressed as

$$
\begin{aligned}
P_{s}^{\prime}{ }_{s}^{R Q A M}(e \mid \eta)= & \eta^{-\frac{1}{2}}\left[\frac{a_{0} p_{0}\left(q_{0}-1\right)}{\sqrt{2 \pi}} e^{-\frac{a_{0}^{2} \eta}{2}}+\frac{b_{0}\left(p_{0}-1\right) q_{0}}{\sqrt{2 \pi}} e^{-\frac{b_{0}^{2} \eta}{2}}\right] \\
& -\frac{a_{0} b_{0} p_{0} q_{0}}{\pi} e^{-\frac{\left(a_{0}^{2}+b_{0}^{2}\right) \eta}{2}} \\
& \times\left[{ }_{1} F_{1}\left(1 ; 1.5 ; \frac{a_{0}^{2} \eta}{2}\right)+{ }_{1} F_{1}\left(1 ; 1.5 ; \frac{b_{0}^{2} \eta}{2}\right)\right] .
\end{aligned}
$$

Substituting $P_{s}^{\prime R Q A M}(e \mid \eta)$ and $P_{\text {out }, L B}(\eta)$ from $(\mathrm{D} .2)$ and $(\mathrm{B} .2)$, respectively in (16) and solving the required integrals with the aid of [48, (3.381.4), (7.522.9)], ASER expression of general order RQAM scheme is expressed as (18).

\section{Appendix E. Proof of Lemma 5}

For 32-XQAM scheme, conditional SEP expression in AWGN channel can be expressed as

$$
P_{s}^{X Q A M}(e \mid \eta)=\frac{1}{8}\left[26 Q(\sqrt{2 \Lambda \eta})+Q(2 \sqrt{\Lambda \eta})-23 Q^{2}(\sqrt{2 \Lambda \eta})\right],
$$

where $\Lambda=48 /(31 M-32)$. The first order derivative of E.1 is

$$
\begin{aligned}
P_{s}^{\prime X Q A M}(e \mid \eta)=-\frac{1}{8} & {\left[\frac{3}{2} \sqrt{\frac{\Lambda}{\pi}} \frac{e^{-\Lambda \eta}}{\sqrt{\eta}}+\sqrt{\frac{\Lambda}{2 \pi}} \frac{e^{-2 \Lambda \eta}}{\sqrt{\eta}}\right.} \\
& \left.+\frac{23 \Lambda}{\pi} e^{-2 \Lambda \eta}{ }_{1} F_{1}\left(1 ; \frac{3}{2} ; \Lambda \eta\right)\right] .
\end{aligned}
$$

From E.2 and $\mathrm{B} .2$, values of $P_{s}^{\prime X Q A M}(e \mid \eta)$ and $P_{\text {out }, L B}(\eta)$, respectively are substituted in 16). Finally, solving the required integrals with the aid of [48. (3.381.4), (7.522.9)], ASER expression of 32-XQAM scheme is expressed as 


\section{Appendix F. Proof of Lemma 6}

Taking the first order derivative of 14 the PDF of the end-to-end instantaneous SNR can be given as

$$
\begin{aligned}
f_{\eta_{s r_{1} r_{2} d}}(\eta) & =Q_{1}\left(\sqrt{2 K_{s r_{1}}}, \sqrt{2 \Omega_{s r_{1}} \eta}\right) e^{\frac{-\eta}{\eta_{r_{1} r_{2}}}} f_{\eta_{r_{2} d}}(\eta) \\
& +Q_{1}\left(\sqrt{2 K_{s r_{1}}}, \sqrt{2 \Omega_{s r_{1}} \eta}\right) f_{\eta_{r_{1} r_{2}}}(\eta) Q_{1}\left(\sqrt{2 K_{r_{2} d}}, \sqrt{2 \Omega_{r_{2} d} \eta}\right) \\
& +f_{\eta_{s r_{1}}}(\eta) e^{\frac{-\eta}{\bar{\eta}_{1} r_{2}}} Q_{1}\left(\sqrt{2 K_{r_{2} d}}, \sqrt{2 \Omega_{r_{2} d} \eta}\right)
\end{aligned}
$$

where $f_{\eta_{(.)}}(\eta)$ represents the PDF of a particular links SNR, expressed in A.2 and (A.4).

By introducing (F.1) in 20, we obtain

$$
\begin{aligned}
C_{o p t} & =\frac{1}{U} \int_{0}^{\infty} \log _{2}(1+\eta)\left[Q_{1}\left(\sqrt{2 K_{s r_{1}}}, \sqrt{2 \Omega_{s r_{1}} \eta}\right) e^{\frac{-\eta}{\eta_{1} r_{2}}} f_{\eta_{r_{2} d}}(\eta)\right. \\
& +Q_{1}\left(\sqrt{2 K_{s r_{1}}}, \sqrt{2 \Omega_{s r_{1}} \eta}\right) f_{\eta_{r_{1} r_{2}}}(\eta) Q_{1}\left(\sqrt{2 K_{r_{2} d}}, \sqrt{2 \Omega_{r_{2} d} \eta}\right) \\
& \left.+f_{\eta_{s r_{1}}}(\eta) e^{\frac{-\eta}{\eta_{1} r_{2}}} Q_{1}\left(\sqrt{2 K_{r_{2} d}}, \sqrt{2 \Omega_{r_{2} d} \eta}\right)\right] d \eta .
\end{aligned}
$$

Due to the mathematical intractability in achieving the solution of (F.2), approximate form of the first order Marcum Q-function and the PDF of the instantaneous SNR of individual links are considered to get the solution of ergodic capacity. Thus, after substituting the approximate form of the Marcum Q-function from (B.1), and the PDF of the instantaneous SNRs of Rayleigh and Rician distributed links from $\mathrm{A} .2$ and $\mathrm{A} .4$, respectively into $\sqrt{\mathrm{F} .2}, C_{\text {opt }}$ can be further expressed as

$$
\begin{aligned}
C_{o p t} & =\frac{e^{-\left(K_{s r_{1}}+K_{r_{2} d}\right)}}{U \log _{e}(2)} \sum_{l=0}^{\infty} \sum_{m=0}^{l} \sum_{l_{1}=0}^{\infty} \frac{1}{m ! l !\left(l_{1}^{2}\right) !}\left[K_{s r_{1}}^{l} K_{r_{2} d}^{l_{1}}\left(\Omega_{s r_{1}}\right)^{m}\left(\Omega_{r_{2} d}\right)^{l_{1}+1}\right. \\
& \left.+K_{s r_{1}}^{l_{1}} K_{r_{2} d}^{l}\left(\Omega_{r_{2} d}\right)^{m}\left(\Omega_{s r_{1}}\right)^{l_{1}+1}\right] \int_{0}^{\infty} \log _{e}(1+\eta) \eta^{m+l_{1}} e^{-\left(\Omega_{s r_{1}}+\frac{1}{\bar{\eta}_{r_{1} r_{2}}}+\Omega_{r_{2} d}\right) \eta} d \eta \\
& +\frac{e^{-\left(K_{s r_{1}}+K_{r_{2} d}\right)}}{U \log _{e}(2)} \frac{1}{\bar{\eta}_{r_{1} r_{2}}} \sum_{l=0}^{\infty} \sum_{m=0}^{l} \sum_{l_{1}=0}^{\infty} \sum_{m_{1}=0}^{l_{1}} \frac{K_{s r_{1}}^{l} K_{r_{2} d}^{l_{1}}}{l !\left(l_{1}\right) ! m ! m_{1} !}\left(\Omega_{s r_{1}}\right)^{m}\left(\Omega_{r_{2} d}\right)^{m_{1}} \\
& \times \int_{0}^{\infty} \log _{e}(1+\eta) \eta^{m+m_{1}} e^{-\left(\Omega_{s r_{1}}+\frac{1}{\bar{\eta}_{1} r_{2}}+\Omega_{r_{2} d}\right) \eta} d \eta .
\end{aligned}
$$

Finally, using the integral from [49, (7)], ergodic capacity is expressed in (21).

\section{Acknowledgment}

The authors would like to thank IIT Indore, and Visvesvarya PhD scheme for all the support. 


\section{References}

[1] D. Raychaudhuri, N. B. Mandayam, Frontiers of wireless and mobile communications, IEEE Proceed. 100 (4) (2012) 824-840.

[2] Q. C. Li, H. Niu, A. T. Papathanassiou, G. Wu, 5G network capacity: key elements and technologies, IEEE Veh. Technol. Mag. 9 (1) (2014) 71-78.

[3] J. N. Laneman, D. N. Tse, G. W. Wornell, Cooperative diversity in wireless networks: Efficient protocols and outage behavior, IEEE Trans. Inform. Theory 50 (12) (2004) 3062-3080.

[4] N. Kumar, V. Bhatia, Exact ASER analysis of rectangular QAM in twoway relaying networks over Nakagami-m fading channels, IEEE Wireless Commun. Lett. 5 (5) (2016) 548-551.

[5] N. Kumar, V. Bhatia, D. Dixit, Performance analysis of QAM in amplifyand-forward cooperative communication networks over Rayleigh fading channels, AEU-Int. J. Electron. Commun. 72 (2017) 86-94.

[6] İ. Altunbaş, A. Yılmaz, Ş. S. Kucur, O. Kucur, Performance analysis of dual-hop fixed-gain AF relaying systems with OSTBC over Nakagami-m fading channels, AEU-Int. J. Electron. Commun. 66 (10) (2012) 841-846.

${ }_{445}^{4}$ [7] Y. Yang, H. Hu, J. Xu, G. Mao, Relay technologies for WiMAX and LTEAdvanced mobile systems, IEEE Commun. Mag. 47 (10) (2009) 100-105.

[8] K. R. Liu, Cooperative Communications and Networking, Cambridge University Press, 2009.

[9] N. Bissias, G. P. Efthymoglou, V. A. Aalo, Performance analysis of dualhop relay systems with single relay selection in composite fading channels, AEU-Int. J. Electron. Commun. 66 (1) (2012) 39-44.

[10] E. Soleimani-Nasab, M. Ardebilipour, A. Kalantari, B. Mahboobi, Performance analysis of multi-antenna relay networks with imperfect channel estimation, AEU-Int. J. Electron. Commun. 67 (1) (2013) 45-57.

455 [11] C. K. Datsikas, K. P. Peppas, F. I. Lazarakis, G. S. Tombras, Error rate performance analysis of dual-hop relaying transmissions over generalized-K fading channels, AEU-Int. J. Electron. Commun. 64 (11) (2010) 1094-1099.

[12] N. Kumar, V. Bhatia, Outage analysis of OFDM AF relaying systems over Nakagami-m fading channels with non-linear power amplifier, in: IEEE Wireless Commun. Netw. Conf. (WCNC), IEEE, 2016, pp. 1-6.

[13] P. K. Singya, N. Kumar, V. Bhatia, Performance analysis of AF OFDM system using multiple relay in presence of nonlinear-PA over inid Nakagamim fading, Wiley Int. J. Commun. Syst. 31 (1) (2018) 1-15.

[14] C. Zhong, M. Matthaiou, G. K. Karagiannidis, A. Huang, Z. Zhang, Capacity bounds for AF dual-hop relaying in $\mathcal{G}$ fading channels, IEEE Trans. Veh. Technol. 61 (4) (2012) 1730-1740. 
[15] N. Yang, M. Elkashlan, J. Yuan, Outage probability of multiuser relay networks in Nakagami- $m$ fading channels, IEEE Trans. Veh. Technol. 59 (5) (2010) 2120-2132.

470 [16] A. F. Molisch, Wireless Communications, John Wiley \& Sons, 2007.

[17] P. K. Singya, N. Kumar, V. Bhatia, Mitigating NLD for wireless networks: Effect of nonlinear power amplifiers on future wireless communication networks, IEEE Microwave Mag. 18 (5) (2017) 73-90.

[18] C. R. Stevenson, G. Chouinard, Z. Lei, W. Hu, S. J. Shellhammer, W. Caldwell, IEEE 802.22: The first cognitive radio wireless regional area network standard, IEEE Commun. Mag. 47 (1) (2009) 130-138.

[19] A. Bishnu, V. Bhatia, Sparse channel estimation for interference limited OFDM systems and its convergence analysis, IEEE Access 5 (2017) 1778117794.

${ }_{480}$ [20] T. Hwang, C. Yang, G. Wu, S. Li, G. Y. Li, OFDM and its wireless applications: a survey, IEEE Trans. Veh. Technol. 58 (4) (2009) 1673-1694.

[21] N. Kumar, V. Bhatia, Performance analysis of OFDM based AF cooperative systems in selection combining receiver over Nakagami-m fading channels with nonlinear power amplifier, Wiley Int. J. Commun. Syst. 30 (7) (2017) 1-17.

[22] G. Berardinelli, K. Pajukoski, E. Lahetkangas, R. Wichman, O. Tirkkonen, P. Mogensen, On the potential of OFDM enhancements as $5 \mathrm{G}$ waveforms, in: IEEE Veh. Technol. Conf. (VTC), IEEE, 2014, pp. 1-5.

[23] D. Zhang, Y. Wang, J. Lu, QoS aware relay selection and subcarrier allocation in cooperative OFDMA systems, IEEE Commun. Lett. 14 (4) (2010) 294-296.

[24] D. Dixit, P. R. Sahu, Performance analysis of rectangular QAM with SC receiver over Nakagami- $m$ fading channels, IEEE Commun. Lett. 18 (7) (2014) 1262-1265.

[25] N. Kumar, P. K. Singya, V. Bhatia, ASER analysis of hexagonal and rectangular QAM schemes in multiple relay networks, IEEE Trans. Veh. Technol. 67 (2) (2018) 1815-1819.

[26] P. K. Singya, N. Kumar, V. Bhatia, Impact of imperfect CSI on ASER of hexagonal and rectangular QAM for AF relaying network, IEEE Commun. Lett. 22 (2) (2018) 428-431.

[27] R. Schober, UE-side virtual MIMO using mm-wave for 5G, IIS Whitepaper.

[28] G. K. Karagiannidis, Performance bounds of multihop wireless communications with blind relays over generalized fading channels, IEEE Trans. Wireless Commun. 5 (3) (2006) 498-503.

${ }_{505}$ [29] H. A. Suraweera, R. H. Louie, Y. Li, G. K. Karagiannidis, B. Vucetic, Two hop amplify-and-forward transmission in mixed Rayleigh and Rician fading channels, IEEE Commun. Lett. 13 (4) (2009) 227-229. 
[30] H. A. Suraweera, G. K. Karagiannidis, P. J. Smith, Performance analysis of the dual-hop asymmetric fading channel, IEEE Trans. Wireless Commun. 8 (6) (2009) 2783-2788.

[31] W. Xu, J. Zhang, P. Zhang, Performance analysis of dual-hop amplify-andforward relay system in mixed Nakagami-m and Rician fading channels, IET Electron. Lett. 46 (17) (2010) 1231-1232.

[32] S. S. Soliman, N. C. Beaulieu, Dual-hop AF relaying systems in mixed Nakagami-m and Rician links, in: IEEE Globecom Workshops (GC Wkshps), IEEE, 2012, pp. 447-452.

[33] A. A. Mohammed, L. Yu, M. Al-Kali, E. E. B. Adam, BER analysis and evaluated for different channel models in wireless cooperation networks based OFDM system, in: IEEE Int. Conf. Commun. Syst. Network Technol. (CSNT), IEEE, 2014, pp. 326-330.

[34] M. Mohammadi, M. Ardebilipour, Z. Mobini, R.-A. S. Zadeh, Performance analysis and power allocation for multi-hop multi-branch amplify-andforward cooperative networks over generalized fading channels, EURASIP J. Wireless Commun. Netw. 2013 (1) (2013) 1-13.

[35] M. R. Bhatnagar, On the capacity of decode-and-forward relaying over Rician fading channels, IEEE Commun. Lett. 17 (6) (2013) 1100-1103.

[36] S. S. Soliman, N. C. Beaulieu, The bottleneck effect of Rician fading in dissimilar dual-hop AF relaying systems, IEEE Trans. Veh. Technol. 63 (4) (2014) 1957-1965.

[37] S. S. Soliman, MRC and selection combining in dual-hop AF systems with Rician fading, in: IEEE Int. Conf. Comput. Eng. \& Syst. (ICCES), IEEE, 2015, pp. 314-320.

[38] L. Rugini, Symbol error probability of hexagonal QAM, IEEE Commun. Lett. 20 (8) (2016) 1523-1526.

${ }_{535}$ [39] S. J. Park, Performance analysis of triangular quadrature amplitude modulation in AWGN channel, IEEE Commun. Lett. 16 (6) (2012) 765-768.

[40] C. A. R. Fernandes, D. B. da Costa, A. L. de Almeida, Performance analysis of cooperative amplify-and-forward orthogonal frequency division multiplexing systems with power amplifier non-linearity, IET Commun. 8 (18) (2014) 3223-3233.

[41] M. K. Simon, M.-S. Alouini, Digital communication over fading channels, Vol. 95, John Wiley \& Sons, 2005.

[42] M. Torabi, J. F. Frigon, D. Haccoun, Adaptive transmission in amplify-andforward cooperative communications using orthogonal space-time block codes under spatially correlated antennas, IET Commun. 9 (14) (2015) $1683-1690$

[43] V. Asghari, D. B. da Costa, S. Aissa, Performance analysis for multihop relaying channels with Nakagami-m fading: Ergodic capacity upper-bounds and outage probability, IEEE Trans. Commun. 60 (10) (2012) 2761-2767. 
${ }_{550}^{50}$ [44] I. Trigui, S. Affes, A. Stephenne, Ergodic capacity analysis for interferencelimited AF multi-hop relaying channels in Nakagami-m fading, IEEE Trans. Commun. 61 (7) (2013) 2726-2734.

[45] A. Behnad, X. Wang, Accuracy of harmonic mean approximation in performance analysis of multihop amplify-and-forward relaying, IEEE Wireless Commun. Lett. 3 (2) (2014) 125-128.

[46] K. Song, Y. Wang, C. Li, Y. Huang, L. Yang, Performance analysis of selforganizing heterogeneous network with interference cancellation, in: IEEE Wireless Commun. Netw. Conf. Workshops (WCNCW), IEEE, 2015, pp. $282-286$.

[47] M. Abramowitz, I. A. Stegun, Handbook of mathematical functions: with formulas, graphs, and mathematical tables, 9th ed. New York, NY, USA: Dover, 1970.

[48] I. Gradshteyn, I. Ryzhik, Table of Integrals, Series and Products, 6th ed. New York, NY, USA: Academic, 2000.

565 [49] C. Gunther, Comment on estimate of channel capacity in Rayleigh fading environment, IEEE Trans. Veh. Technol. 45 (2) (1996) 401-403. 